\title{
Reactive chemical dynamics through conical intersections
}

\author{
S GHOSAL, B JAYACHANDER RAO and S MAHAPATRA* \\ School of Chemistry, University of Hyderabad, Hyderabad 500046 \\ e-mail:smsc@uohyd.ernet.in
}

MS received 22 June 2007; accepted 31 July 2007

\begin{abstract}
Reaction dynamics of prototypical, $\mathrm{D}+\mathrm{H}_{2}$ and $\mathrm{Cl}\left({ }^{2} \mathrm{P}\right)+\mathrm{H}_{2}$, chemical reactions occurring through the conical intersections of the respective coupled multi-sheeted potential energy surfaces is examined here. In addition to the electronic coupling, nonadiabatic effects due to relativistic spin-orbit coupling are also considered for the latter reaction. A time-dependent wave packet propagation approach is undertaken and the quantum dynamical observables viz., energy resolved reaction probabilities, integral reaction cross-sections and thermal rate constants are reported.
\end{abstract}

Keywords. Time-dependent quantum wave packet dynamics; conical intersections; nonadiabatic coupling; spin-orbit coupling.

\section{Introduction}

In recent years non-adiabatic effects due to potential energy surface (PES) crossings and spin-orbit (SO) coupling have been realized to play crucial role in the dynamics of chemical reactions. Attention is therefore paid by various research groups to devise accurate quantum mechanical model in order to simulate chemical reaction dynamics on multi-sheeted coupled PESs to understand and interpret the modern experimental molecular beam data. ${ }^{1}$ In the recent past dynamical calculations of reaction cross-sections and thermal rate constants, relied on a single (adiabatic) ground PES of the reaction system. ${ }^{2}$ However, recent experimental advances on the reactivity of the ground and excited electronic states of many chemical reactions unfolds the role of non-adiabatic coupling (NAC) between different closely spaced electronic states and such a single-surface theoretical study appears to be inadequate. ${ }^{3}$ Full-dimensional quantum dynamical calculations are necessary considering all the interacting electronic states which correlate with the reagent asymptote as well as all the NAC between them in order to investigate such experimental data.

In this article, we consider two prototypical chemical reactions, e.g. $\mathrm{D}+\mathrm{H}_{2}$ and $\mathrm{Cl}\left({ }^{2} \mathrm{P}\right)+\mathrm{H}_{2}$ and study the importance of NAC in their nuclear dynamics. Both the reactions occur through the conical intersections $(\mathrm{CIs})^{3}$ of their respective PESs. The $\mathrm{D}+\mathrm{H}_{2}$ exchange

\footnotetext{
*For correspondence
}

reaction takes place on a doubly degenerate ground electronic state which splits upon distortion from the equilibrium configuration of $\mathrm{DH}_{2}$. The resulting two component PESs form CIs at the equilibrium configuration of the undistorted geometry.

The dynamics of $\mathrm{D}+\mathrm{H}_{2}$ reaction has been studied by Gray and his coworkers ${ }^{4}$ to calculate the reaction probabilities for the total angular momentum, $J=0$ on the Liu-Siegbahn-Turhlar-Horowitz (LSTH) ${ }^{5}$ potential energy surface. Recently, Sukiasyan et al have calculated the initial state-selected reaction cross-sections for this reaction using the same LSTH PES for the collision energies up to $1.3 \mathrm{eV}$. These are single surface calculations, done on the repulsive adiabatic sheet of the ground electronic state of the system. The effect of surface coupling on the dynamics of this reaction has been studied by including the geometric phase change of the electronic wave function on the latter surface. ${ }^{7,8}$ None of the previous calculations have considered both the surfaces explicitly and the nonadiabatic coupling between them. Our study here includes both the sheets of the degenerate ground electronic states and their coupling surfaces. We have employed a time-dependent wave packet (TDWP) method that is previously developed to calculate the initial state-selected total reaction probability for the $\mathrm{H}+\mathrm{H}_{2}{ }^{9-11}$ The calculations for the non-zero total angular momentum are carried out within the centrifugal sudden or coupled states (CS) approximation. The reaction probabilities, integral reaction crosssections and thermal rate constants are reported. 
The reactions between halogen atoms and molecular hydrogen represent prototypical examples to study and understand the effect of spin orbit (SO) coupling in the dynamics. ${ }^{12}$ The approach of the $\mathrm{H}_{2}$ molecule to the $\mathrm{Cl}$ atom splits the threefold degeneracy of the ${ }^{2} \mathrm{P}$ state and leads to three adiabatic electronic states with ${ }^{2} \sum_{1 / 2},{ }^{2} \prod_{3 / 2}$ and ${ }^{2} \prod_{1 / 2}$ symmetries in collinear geometries $\left(1^{2} \mathrm{~A}^{\prime}, 1{ }^{2} \mathrm{~A}^{\prime \prime}\right.$ and $2^{2} \mathrm{~A}^{\prime}$ symmetries, respectively, in non-collinear geometries) (cf. figure 1 of ref. 13). In reagent arrangement, the first two states correspond to the ground ${ }^{2} \mathrm{P}_{3 / 2} \mathrm{SO}$ state of the atomic $\mathrm{Cl}$, and the third state correlates to its excited ${ }^{2} \mathrm{P}_{1 / 2}$ SO state. The two states of ${ }^{2} \mathrm{~A}^{\prime}$ symmetry form CIs in the collinear geometry. In the product arrangement, the ${ }^{2} \Sigma_{1 / 2}$ adiabatic state correlates with the electronic ground state of the products, whereas the other two states correlate with the electronically excited products. The latter two states are adiabatically closed at low and moderate collision energies, but they may yield products in their electronic ground state via nonadiabatic transitions to the ${ }^{2} \sum_{1 / 2}$ electronic state. ${ }^{14}$

Recently, we have studied the dynamics of the $\mathrm{Cl}$ $\left({ }^{2} \mathrm{P}\right)+\mathrm{H}_{2}$ reaction for the total angular momentum $J=0 \cdot 5$, by considering the electronic and $\mathrm{SO}$ coupling. ${ }^{15}$ We here extended this study to calculate the reaction probabilities for all non-zero values of $J$ within the CS approximation. The reaction crosssections and the thermal rate constants are obtained from the calculated reaction probabilities in both uncoupled and coupled surface situations and the results are compared with the available theoretical and experimental results. ${ }^{16-19}$ Here we note that, the uncoupled surface results reported in this paper refer to the treatment of the nuclear dynamics on the lower adiabatic sheet only without including any non-adiabatic coupling in the dynamics. The rest of the paper is organized in the following way; in $\S 2$, we present the theoretical formalism employed for this work. The results for the $\mathrm{D}+\mathrm{H}_{2}$ and $\mathrm{Cl}\left({ }^{2} \mathrm{P}\right)+\mathrm{H}_{2}$ reactions are presented and discussed in $\S 3$. A summary of our findings are presented in $\S 4$.

\section{Theory}

We have employed a TDWP ${ }^{20}$ approach to calculate the dynamical observables, e.g. the initial state-selected and energy resolved reaction probabilities, integral reaction cross-sections and thermal rate constants. The reaction probabilities are calculated from the expectation values of the quantum flux operator on the basis of energy normalized time-independent reactive scattering wave function. The initial wave packet
(WP), $|\psi(t=0)\rangle$, pertaining to the reagents is prepared in the asymptotic reagent channel where there is no interaction between the reagent atom and diatom. This WP is propagated in the coupled manifold of electronic states of the respective system by solving the time-dependent Schrödinger equation (TDSE) numerically on a grid. For an explicitly timeindependent Hamiltonian the solution reads

$$
|\psi(t)\rangle=\exp \left[\frac{-i \hat{H} t}{\hbar}\right]|\psi(t=0)\rangle,
$$

where $|\psi(t)\rangle$ is the wave function of the reacting system at time $t$, and $\hat{H}$ defines the Hamiltonian operator of the collisional system. The initial wave function, $|\psi(t=0)\rangle$ in the asymptotic reactant channel can be represented as a product of the translational wave function for the approach of the atom to the diatom and the rovibrational wavefunction of the diatomic reagent molecule (here $\mathrm{H}_{2}$ ).

For the $\mathrm{D}+\mathrm{H}_{2}$ system, the reaction occurs on $2 \times 2$ coupled electronic states. Therefore, the interaction Hamiltonian in a diabatic electronic basis can be written as

$$
H^{d}=T_{N}\left(\begin{array}{ll}
1 & 0 \\
0 & 1
\end{array}\right)+\left(\begin{array}{ll}
U_{11} & U_{12} \\
U_{21} & U_{22}
\end{array}\right),
$$

where $T_{N}$ represents the diagonal nuclear kinetic energy operator and the second term represents the nondiagonal electronic part of the Hamiltonian in the diabatic electronic basis. In terms of the mass-scaled body-fixed (BF) Jacobi coordinates $R, r$ and $\gamma$ (symbols representing their usual meaning) and for the total angular momentum $J \neq 0, T_{N}$ is given by

$$
T_{N}=-\frac{\hbar}{2 \mu}\left[\frac{\partial^{2}}{\partial R^{2}}+\frac{\partial^{2}}{\partial r^{2}}\right]+\frac{\hat{j}}{2 \mu r^{2}}+\frac{\hat{l}}{2 \mu R^{2}} .
$$

The quantities $U_{11}$ and $U_{22}$ in (2) are the energies of the two diabatic electronic states and, $U_{12}=U_{21}$, represent the electronic coupling between them. The operator $\hat{j}$ defines the diatomic rotational angular momentum and $\hat{l}$ is the orbital angular momentum operator. The quantity, $\mu$ represents the three-body reduced mass calculated accordingly from the masses of the three atoms involved in the reaction. The BF $z$-axis is defined to be parallel to $R$ and the diatom lies in the $(x, z)$ plane. Within the CS approximation $^{21}$ the quantity $\hat{j}_{z}$ is expressed as

$$
\hat{l}^{2} \equiv(\hat{J}-\hat{j})^{2}=\hat{J}^{2}+\hat{j}^{2}-2 \hat{j}_{Z},
$$


where $\hat{J}$ is the total angular momentum operator and $\hat{J}_{z}$ and $\hat{j}_{z}$ are the respective BF $z$ components of $\hat{J}$ and $\hat{j}$. The elements of the diabatic electronic Hamiltonian matrix of (2) are obtained by diabatizing the adiabatic electronic Hamiltonian matrix through a similarity transformation using a unitary adiabaticto-diabatic transformation matrix $(S)$. The two adiabatic sheets of the double many body expansion (DMBE) potential energy surface developed by Varandas $e t a l^{22}$ are diabatized ${ }^{9,10,23}$ and used in the present calculations.

The inclusion of SO coupling in the dynamics of $\mathrm{Cl}\left({ }^{2} \mathrm{P}\right)+\mathrm{H}_{2}$ reaction, on the other hand leads to a problem involving six coupled electronic states. ${ }^{14}$ This can be reduced to a three states problem in a suitable complex basis representation. ${ }^{24}$ In a diabatic electronic basis, the nuclear part of the Hamiltonian becomes a $3 \times 3$ diagonal matrix and the non-diagonal electronic part of the Hamiltonian involves six potential energy surfaces, ${ }^{24}$ three diabatic PESs $\left(V_{\Sigma}, V_{\Pi}\right.$ and $\left.V_{2}\right)$, one electronic coupling surface $\left(V_{1}\right)$, and two SO coupling surfaces $(A$ and $B)$. The explicit form of the electronic Hamiltonian matrix becomes

$$
H=\left(\begin{array}{ccc}
V_{\Sigma} & -V_{1}-i \sqrt{2} B & V_{1} \\
-V_{1}+i \sqrt{2} B & V_{\Pi}+A & V_{2} \\
V_{1} & V_{2} & V_{\Pi}-A
\end{array}\right) .
$$

The elements of the above six PESs are calculated by Capecchi and Werner employing the state-of-the-art $a b$ initio methods. ${ }^{14}$ We use their PESs in our dynamical studies below.

The reaction probability is obtained from the expectation value of the flux operator in the basis of energy normalized reactive scattering wave function. This is calculated in the asymptotic product channel and on a dividing surface which separates the products from the reactants. The energy normalized wave functions are obtained by Fourier transforming the time-evolved WP along the divide surface. The explicit expression for the state-selected and energy resolved reaction probability in a diabatic electronic representation, is given by ${ }^{9}$

$$
\begin{aligned}
P_{v j}^{R}(E) & =\frac{h}{2 \pi \mu} \sum_{k=1}^{n} \operatorname{Im} \\
& {\left[\left\langle\phi_{k}^{d}\left(R, r_{d}, \gamma, E\right)|| \frac{\partial \phi_{k}^{d}\left(R, r_{d}, \gamma, E\right)}{\partial r}\right\rangle\right]_{r=r_{d}}, }
\end{aligned}
$$

where, the summation runs over all diabatic electronic states involved in the reaction dynamics. The quantity on the right hand side of (6) is integrated over the entire range of $R$ and $\gamma$ to obtain the reaction probability starting from a given vibrational and rotational $(v, j)$ state of the reagent diatom.

In the adiabatic electronic representation the electronic part of the Hamiltonian is diagonal and the non-adiabatic coupling elements appear as off-diagonal elements in the nuclear Hamiltonian. Hence the flux operator in the adiabatic electronic basis possesses off-diagonal elements. Consequently, the probability expression in the adiabatic electronic basis, contains the off-diagonal electronic contributions in contrast to the same in the diabatic electronic representation (for details please see ref. 25 and references therein).

The cumulative reaction probability for a given initial state $(v, j)$ is calculated by summing up the reaction probability results for different partial wave contributions of the total angular momentum $J$. The total reaction cross-section is calculated from the reaction probability as ${ }^{26}$

$$
\sigma_{v j}(E)=\frac{\pi}{\kappa^{2}} \sum_{\Omega=0}^{j} \frac{g_{\Omega}}{(2 j+1)} \sum_{J \geq \Omega}^{J_{\max }}(2 J+1) P_{v j}^{J \Omega}(E) .
$$

where the quantity $g_{\Omega}$ is the degeneracy factor $\left(g_{\Omega}=1\right.$ for $\Omega=0$, and $g_{\Omega}=2$ for $\Omega \neq 0, \Omega$ being the projection quantum number of the total angular momentum $J$ ) and $\kappa$ is the weight of the translational component of the initial WP.

The initial state-selected thermal rate constant ${ }^{27}$ can be obtained from the total reaction cross-section as

$$
k_{v j}(T)=\sqrt{\frac{8 k_{B} T}{\pi \mu}} \frac{1}{\left(k_{B} T\right)^{2}} \int_{0}^{\infty} E \sigma_{v j}(E) e^{-E / k_{B} T} d E,
$$

where $k_{B}$ is the Boltzmann constant. Finally, the rotationally averaged thermal rate constant can be obtained by averaging over a Boltzmann distribution of such states

$$
k_{v}(T)=\sum_{j} \frac{k_{v j}(T)}{Q_{r o t}}(2 j+1) e^{-B j(j+1) h c / k_{B} T},
$$

where $B$ is the rotational constant of the reagent and $Q_{\text {rot }}$ is the rotational partition function.

\section{Results and discussion}

The $\mathrm{D}+\mathrm{H}_{2}(v=0, j=0) \rightarrow \mathrm{HD}\left(\Sigma v^{\prime}, \Sigma j^{\prime}\right)+\mathrm{H}$ reaction probability values as a function of the total energy $E$ 
(D, $\mathrm{H}_{2}$ translational $+\mathrm{H}_{2}$ rovibrational) are plotted in figure 1 for a few selected values of the total angular momentum, $J=0,10,20,30,40$ and 50 (indicated in the panel) and for $\Omega=0$. The coupled and uncoupled surface results are shown by the solid and dashed lines, respectively. The coupled surface results here refer to those obtained by simulating the nuclear dynamics using the Hamiltonian of (2) and the uncoupled

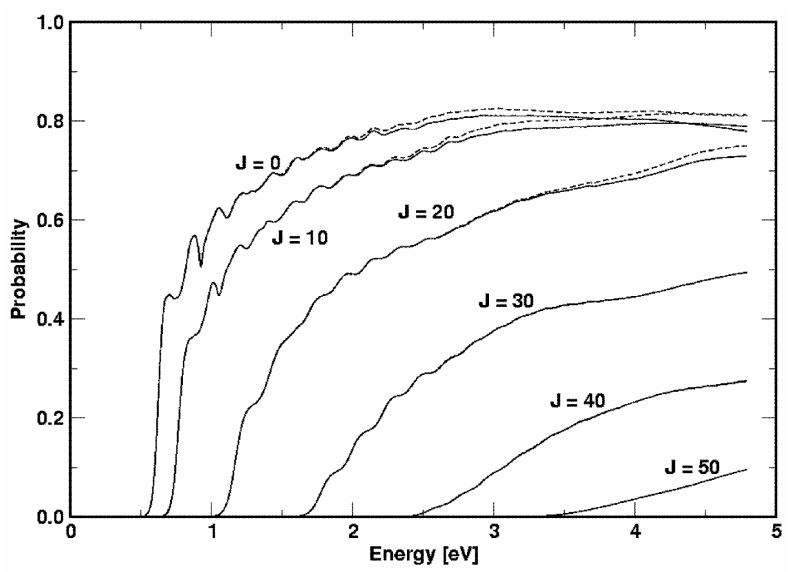

Figure 1. Total reaction probabilities as a function of the total energy $E$ for the $\mathrm{D}+\mathrm{H}_{2}(v=0, j=0) \rightarrow \mathrm{HD}$ $\left(\Sigma v^{\prime}, \Sigma j^{\prime}\right)+\mathrm{H}$ exchange reaction for the total angular momentum, $J=0,10,20,30,40$ and 50. The coupled and uncoupled surface results are shown by the solid and dashed lines, respectively.

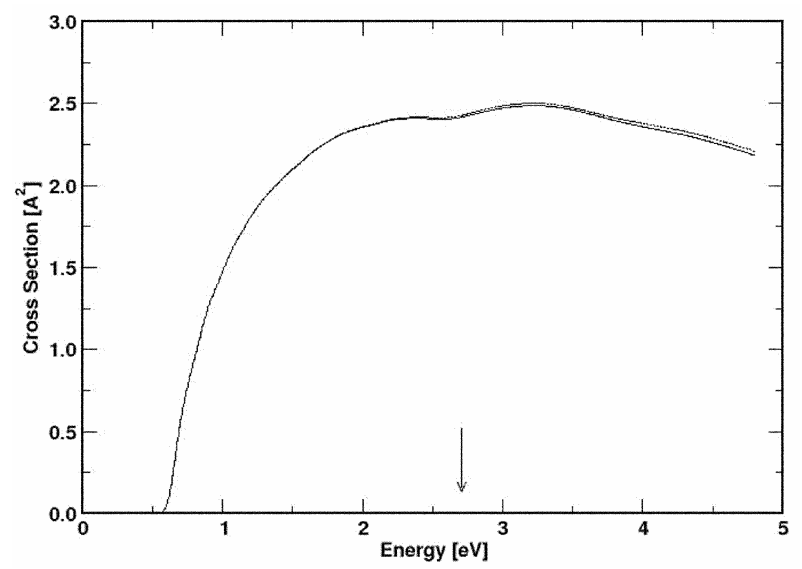

Figure 2. Initial state-selected integral cross-sections of the $\mathrm{D}+\mathrm{H}_{2}(v=0, j=0)$ reaction as a function of the total energy. The coupled and uncoupled surface results are shown by the solid and dashed lines, respectively. The energetic location of the minimum of the seam of conical intersection occurring at $\sim 2.74 \mathrm{eV}$ is indicated by an arrow in the abscissa. surface results are those obtained by simulating the nuclear dynamics on the uncoupled lower adiabatic sheet of the DMBE PES. ${ }^{5}$ The reaction probabilities are calculated up to the three-body dissociation limit of the $\mathrm{DH}_{2}$ system $(\sim 4 \cdot 7 \mathrm{eV})$. As $J$ increases, the reaction threshold increases to higher energy values due to an increase in the centrifugal barrier height with $J$. The resonance structures and their energetic locations remain same in both coupled and uncoupled surface results for a given value of $J$. The difference between the coupled and the uncoupled surface reaction probabilities for a fixed value of $J$ are nearly zero at low energies. At higher energies near and above the minimum of the CIs, this difference becomes noticeable. With increase in $J$, this difference becomes insignificant.

The initial state-selected and energy resolved integral reaction cross-sections of $\mathrm{D}+\mathrm{H}_{2}(v=0, j=0)$ reaction are plotted as a function of the total energy and are shown in figure 2 . The coupled and uncoupled surface results are shown by the solid and dashed lines, respectively. It can be seen from figure 2 that the reaction cross-section increases with energy reaching a maximum value in the energy range of $\sim 2.5-3.5 \mathrm{eV}$ and then decreases. The difference between the coupled and uncoupled surface results is small at low energies and increases very slightly beyond the energetic minimum of the seam of CIs, occurring at $\sim 2.74 \mathrm{eV}$.

The thermal rate constants obtained for the $\mathrm{D}+\mathrm{H}_{2}(v=0, j=0)$ reaction are shown in figure 3 . The coupled and uncoupled surface results are shown by the solid and dashed lines, respectively. Similar

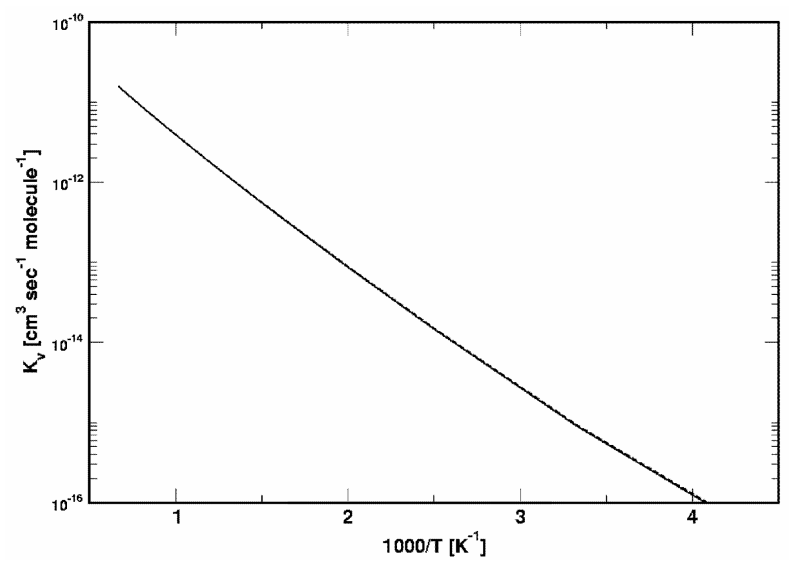

Figure 3. Thermal rate constants for the $\mathrm{D}+\mathrm{H}_{2}(v=0$, $j=0$ ) reaction. The coupled and uncoupled surface results are shown by solid and dashed lines, respectively. 
to the reaction cross-section, the difference between the coupled and uncoupled surface rate constant results is also very small signifying that the $\mathrm{D}+\mathrm{H}_{2}$ $(v=0, j=0)$ reaction dynamics is insensitive to the PES crossings starting from the onset up to the three body dissociation limit.

We now discuss the reaction probability, reaction cross-section and thermal rate constant results obtained for the $\mathrm{Cl}\left({ }^{2} \mathrm{P}\right)+\mathrm{H}_{2}(v=0, j=0) \rightarrow \mathrm{HCl}\left(\Sigma v^{\prime}\right.$, $\left.\Sigma j^{\prime}\right)+\mathrm{H}$ reaction within the CS approximation. To obtain these results, all required WP calculations are carried out in the diabatic electronic representation and by treating $\Omega$ as a fixed parameter. The reaction probabilities are calculated up to a total energy of $1.4 \mathrm{eV}$ and inclusion of partial-wave contribution up to $J=58$ are found to be necessary to obtain converged reaction cross-sections in this energy range. The total reaction probabilities on the uncoupled ${ }^{2} \Sigma$ surface of $\mathrm{Cl}\left({ }^{2} \mathrm{P}\right)+\mathrm{H}_{2}(v=0, j=0)$ reaction as a function of the total energy $E$ are plotted in figure 4 (solid lines) for a few selected values of the total angular momentum $J=0,10,20,40$ and 50 and for $\Omega=0$. It can be seen that the reaction onset shifts to higher energies with an increase in the total angular momentum, $J$. The sharp resonance structures in the reaction probability for $J=0$ are found to become less pronounced with increasing $J$ value, revealing a more direct nature of the reaction. On the average the reaction probabilities for higher values of $J$ are smaller than the corresponding $J=0$ results, indicating substantial non-reactive scattering for $J \neq 0$ collisions.

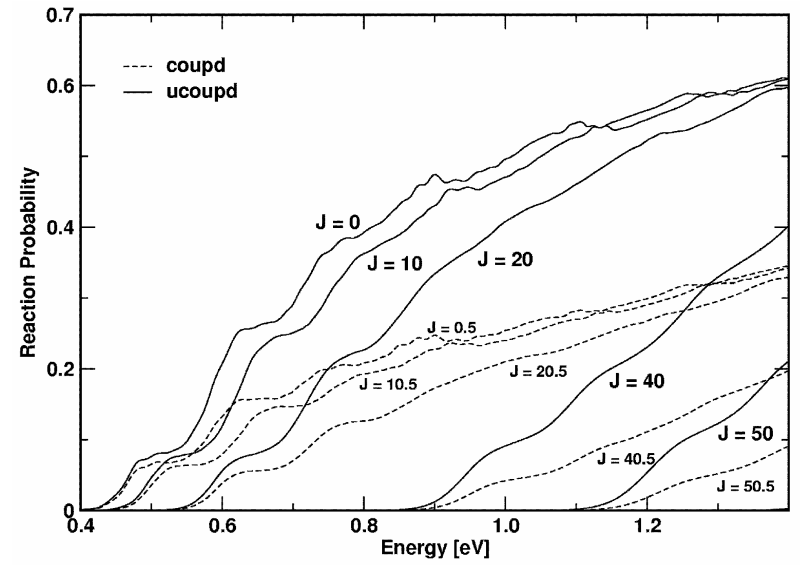

Figure 4. Same as in figure 1 , for the $\mathrm{Cl}\left({ }^{2} \mathrm{P}\right)+\mathrm{H}_{2}$ $(v=0, j=0)$ reaction, for various values of the total angular momentum, $J$ (indicated in the panel). The uncoupled and coupled surface results are shown by the solid and dashed lines, respectively.
In order to examine the effect of the electronic and SO coupling on the above reaction probabilities, we have shown in figure 4 (dashed lines) the energy dependence of the total reaction probability for $J=0.5,10 \cdot 5,20 \cdot 5,40.5$ and 50.5 and for $\Omega=0.5$ obtained in the coupled surface situation (using the Hamiltonian of (5)). In this case the WP is initially located on the reagent asymptote of the ${ }^{2} \Sigma_{1 / 2}$ diabatic surface. The reaction probability significantly decreases (when compared to the uncoupled surface results) with the inclusion of the electronic and $\mathrm{SO}$ coupling in the reaction dynamics. The difference between the uncoupled and coupled surface results also gradually decreases as $J$ increases. Here we note that, the results obtained with the electronic coupling alone do not differ much from the uncoupled surface results, hence we do not show them here. The reaction onset in the coupled surface situation shifts more towards higher energy compared to the uncoupled ones. This is due to an increase in the centrifugal barrier height when SO coupling is included. The resonance structures also disappear with increasing $J$ in the coupled surface situation.

The initial state-selected and energy resolved integral reaction cross-sections of the $\mathrm{Cl}\left({ }^{2} \mathrm{P}\right)+\mathrm{H}_{2}$ $(v=0, j=0)$ reaction, as a function of the total energy are shown in figure 5 . These cross-sections are calculated by summing up different partial wave contributions with appropriate weights to the reaction probability. The uncoupled and coupled (both electronic and SO) surface results are shown by the solid and dashed lines, respectively. The reaction cross-

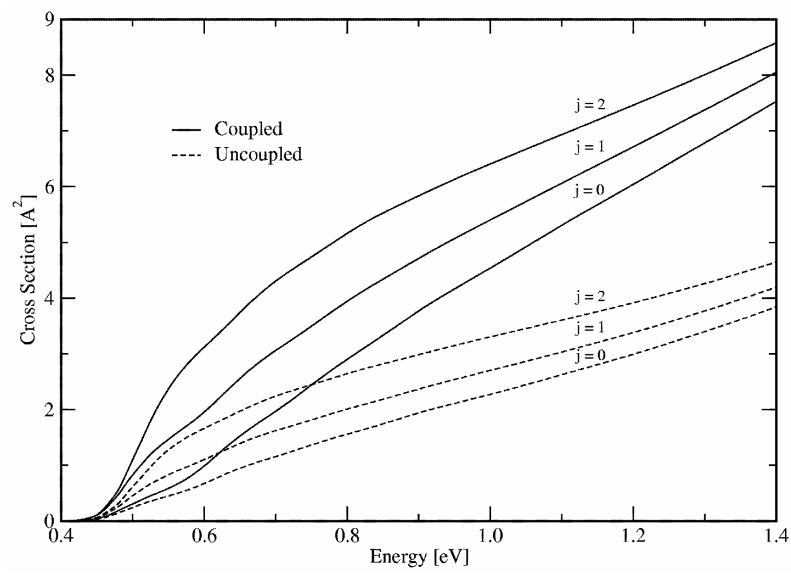

Figure 5. Same as in figure 2, for the $\mathrm{Cl}\left({ }^{2} \mathrm{P}\right)+\mathrm{H}_{2}$ reaction $(v=0, j=0$ to 2$)$. The cross-sections obtained in the uncoupled and coupled surface situations are shown by the solid and dashed lines, respectively. 


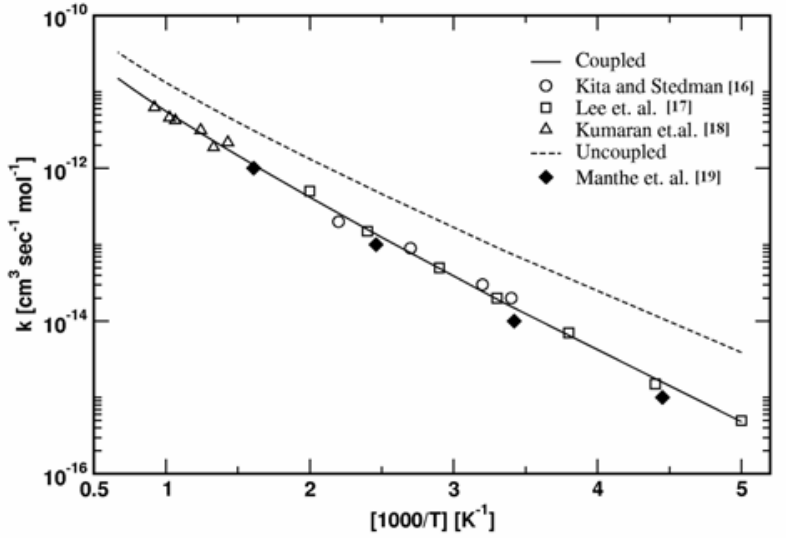

Figure 6. Same as in figure 3 , for the $\mathrm{Cl}\left({ }^{2} \mathrm{P}\right)+\mathrm{H}_{2}$ reaction. Various points on the diagram represent the result form the literature.

sections obtained with rotationally excited reagent $\mathrm{H}_{2}(j=1$ to 2$)$ in its vibrational ground level $(v=0)$ in both uncoupled and coupled surface situations are also shown in the panel. The cross-section results for $j>0$ includes contribution from $\Omega>0$, up to a maximum value of $\Omega_{\max }=\min (j, J)$, within the CS approximation. It can be seen that the reaction crosssection gradually increases with the total energy in both the coupled and uncoupled surface situations and it also increases with increasing rotational excitation of the reagent $\mathrm{H}_{2}$.

The thermal rate constants obtained by statistically averaging over the rotational states $j=0$ to 2 are shown in figure 6 for the $\mathrm{Cl}\left({ }^{2} \mathrm{P}\right)+\mathrm{H}_{2}(v=0)$ reaction. The result obtained by considering the electronic and SO coupling in the dynamics is shown by the solid line and the same without any coupling in the dynamics is represented by the dashed line. The available experimental ${ }^{16-18}$ and recent theoretical results $^{19}$ are shown as different points in the diagram. It can be seen that the thermal rate constants calculated without including any coupling in the dynamics is far from the experimental results whereas the results obtained including the electronic and SO coupling agree well with the experiment. The theoretical rate constants calculated by Manthe et $a l^{19}$ using Multi-configuration time-dependent Hatree approach also uses the SO corrected adiabatic ${ }^{2} \Sigma_{1 / 2}$ PES in the dynamics.

\section{Conclusions}

We have presented a brief theoretical account of the electronic non-adiabatic coupling effects on the dy- namics of $\mathrm{D}+\mathrm{H}_{2}$ and $\mathrm{Cl}\left({ }^{2} \mathrm{P}\right)+\mathrm{H}_{2}$ reactions. The $\mathrm{D}+\mathrm{H}_{2}$ reaction occurs on the lower adiabatic sheet of its coupled ground electronic manifold whereas, the $\mathrm{Cl}\left({ }^{2} \mathrm{P}\right)+\mathrm{H}_{2}$ reaction occurs in the coupled manifold of three electronic states. In addition to the electronic coupling, the relativistic SO coupling is also important in the dynamics of the latter reaction. The quantum dynamical simulations are carried out on a suitable diabatic electronic basis and by a time-dependent WP propagation approach within the CS approximation. Calculation of the dynamical quantities, e.g. initial state-selected energy resolved total reaction probabilities, integral reaction cross-sections and thermal rate constants are carried out both in the uncoupled and coupled surface situations. The NAC has a minor impact on the dynamics of the $\mathrm{D}+\mathrm{H}_{2}$ exchange reaction but a huge impact of the SO coupling on the of $\mathrm{Cl}\left({ }^{2} \mathrm{P}\right)+\mathrm{H}_{2}$ reaction is observed.

\section{Acknowledgement}

This study is supported in part by Council of Scientific and Industrial Research (CSIR), New Delhi. We acknowledge the University Grants Commission (UGC) and CSIR for research fellowships. We also thank UGC (the UPE program) and the Department of Science and Technology, New Delhi (HPCF program) for the computational facilities provided at the University of Hyderabad.

\section{References}

1. Yang X and Liu K (eds) 2004 Modern trends in chemical reaction dynamics: Experiment and theory Part I (Singapore: World-Scientific)

2. Althorpe S C and Clary D C 2003 Annu. Rev. Phys. Chem. 46169

3. Domcke W Yarkony and D R Köppel H (eds) 2004 Conical intersections: Electronic structure dynamics and spectroscopy (Singapore: World-Scientific)

4. Gray S K and Baliant-Kurti G G 1998 J. Chem. Phys. 108950

5. Siegbahn P and Liu B 1978 J. Chem. Phys. 68 2457; Truhlar D G Horowitz C J 1978 J. Chem. Phys. 68 2466; Truhlar D G Horowitz C J 1979 J. Chem. Phys. 711514

6. Sukiasyan S and Meyer H-D 2001 J. Phys. Chem. 1052604

7. Kendrick B K 2000 J. Chem. Phys. 112 5679; Kendrick B K 2003 J. Phys. Chem. A107 6739; Kendrick B K 2003 J. Chem. Phys. 11810502

8. Juanes-Marcos J C and Althorpe S C 2005 J. Chem. Phys. 122 204324; Juanes-Marcos J C, Althorpe S C and Erede E 2005 Science (Lodnon) 3091227 
9. Mahapatra S, Köppel H and Cederbaum L S $2001 \mathrm{~J}$. Phys. Chem. A105 2321

10. Jayachander Rao B, Padmanaban R and Mahapatra S 2007 Chem. Phys. 333135

11. Mahapatra $S$ in ref 3 p. 555

12. Allison T C, Mielke S L, Schwenke D W, Lynch G $\mathrm{C}$, Gordon M S and Truhlar D G Gas-phase reaction systems: experiments and models 100 years after Max Bodenstein (eds) H-R Volp, J Wolfrum R Rannacher and J Warnatz (Springer Heidelburg) p. 111

13. Ghosal S and Mahapatra S 2005 J. Phys. Chem. A109 1530

14. Capecchi G and Werner H-J 2004 Phys. Chem. Chem. Phys. 64975

15. Ghosal S and Mahapatra S 2004 J. Chem. Phys. 121 5740

16. Kita D and Stedman D H 1982 J. Chem. Soc. Faraday Trans. 781249

17. Lee J A, Michael J V, Payne W A and Stief L J 1977 J. Chem. Soc. Faraday Trans 1731530

18. Kumaran S S, Lim K P and Michael J V $1994 J$. Chem. Phys. 1019487
19. Manthe U, Capecchi G and Werner H-J 2004 Phys. Chem. Chem. Phys. 65026

20. Balakrishanan N, Kalyanaraman C and Sathyamurthy N 1997 Phys. Rep. 28079 and references therein

21. Pack R T 1974 J. Chem. Phys. 60 633; McGuire P and Kouri D J 1974 J. Chem. Phys. 602488

22. Varandas A J C, Brown F B, Mead C A, Truhlar D G and Blais N C 1987 J. Chem. Phys. 866258

23. Mahapatra S and Köppel H 1998 Phys. Rev. Lett. 81 3116; Mahapatra S and Köppel H $1998 \mathrm{~J}$. Chem. Phys. 109 1721; Jayachander Rao B, Mahapatra S, Köppel H and Jungen M 2005 J. Chem. Phys. 123 134325

24. Alexander M H, Manolopoulos D E and Werner H-J 2000 J. Chem. Phys. 11311084

25. Köppel H, Domcke W and Cederbaum L S $1984 A d v$. Chem. Phys. 5759

26. Zhang J Z H (ed.) 1999 Theory and application of quantum molecular dynamics (Singapore: WorldScientific)

27. Aoiz F J, Baňares L and Castillo J F 1999 J. Chem. Phys. 1114013 